\title{
Servicehelfer im Sozial- und Gesundheitswesen
}

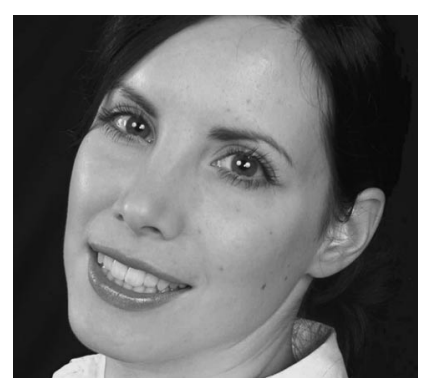

VON MELANIE SCHUSTER Schuster ist Germanistin und Romanistin. Nach verschiedenen Aufgaben im Kulturbereich in Deutschland und Italien koordinierte sie im Auftrag der Robert Bosch Stiftung von Juni 2007 bis September 2009 das Projekt "Servicehelfer im ozial- und Gesundheitswesen «. Internet http://www.boschstiftung.de/servicehelfer
Gemeinsam mit zehn Trägern der Altenund Behindertenhilfe sowie der Krankenpflege aus dem Großraum Stuttgart, Reutlingen und Augsburg hat die Robert Bosch Stiftung das Modellprojekt "Servicehelfer im Sozial- und Gesundheitswesen « entwickelt. Hauptschulabsolventen, die geringere Chancen für einen erfolgreichen Ausbildungs- und Berufsstart mitbringen, werden seit November 2007 in Einrichtungen der Alten- und Behindertenhilfe sowie im Krankenhaus ausgebildet. Sie erbringen Dienstleistungen und Servicetätigkeiten und unterstützen damit Bewohner und Patienten in ihrem Alltag. Die Ausbildung ist sowohl auf die Bedürfnisse der Jugendlichen zugeschnitten als auch speziell auf die Anforderungen der Praxis ausgerichtet.

Das Modellprojekt » Servicehelfer im Sozialund Gesundheitswesen « wurde von Anfang an in enger Abstimmung mit den zehn beteiligten Trägern konzipiert, die sich hierfür zu einem Kooperationsverbund zusammengeschlossen haben. Alle Träger konnten ihre Wünsche an das Aufgabenprofil und an die Curriculumsinhalte einbringen. Damit sollte garantiert werden, dass die Jugendlichen eine Ausbildung erhalten, die auf dem Markt auch nachgefragt wird. Die Evaluationsergebnisse bestätigen, dass sich diese enge konzeptionelle Einbindung der Praxis gelohnt hat. So entspricht das gewählte Ausbildungsprofil aus Sicht aller befragten Einrichtungen, die zum Ende des Ausbildungsjahres des ersten Jahrgangs Servicehelfer ausbildeten, den relevanten Anforderungen an Assistenzkräfte in den Unternehmen. Weiterhin zeigen die Evaluationsergebnisse, dass die Mehrheit der Einrichtungen einen Bedarf an Servicehelfern feststellt.

Die angehenden Servicehelfer werden ein Jahr in Theorie $(30 \%$, im Bildungszentrum des Wohlfahrtswerks für BadenWürttemberg in Stuttgart) und Praxis $(70 \%$, in den beteiligten Einrichtungen) ausgebildet. In den praktischen Einrichtungen der Alten- und Behindertenhilfe sowie im Krankenhaus übernehmen sie Servicetätigkeiten im patienten- und bewohnernahen, hauswirtschaftlichen, (haus-) technischen, logistischen und administrativen Bereich. Es geht nicht um die Übernahme von pflegerischen Tätigkeiten.

\section{Was Servicehelfer tun}

Servicehelfer, die Dienstleistungen im patienten- oder bewohnernahen Bereich durchführen, gehen beispielsweise mit $\mathrm{Pa}$ tienten und Bewohnern spazieren, begleiten diese zu Veranstaltungen, Arztbesuchen oder bei Einkäufen, lesen vor oder helfen bei der Auswahl von Speisen. $\mathrm{Zu}$ den serviceorientierten und hauswirtschaftlichen Tätigkeiten gehören das Reinigen von Rollstühlen, das Betten machen, das Zubereiten von kleineren Mahlzeiten oder Kaffee, Tische decken, Wäsche verteilen oder die Mithilfe in der Cafeteria. Servicehelfer, die Dienstleistungen im (haus-) technischen oder logistischen Bereich durchführen, bringen u. a. Material ins Labor, hängen Bilder auf, wechseln kaputte Glühbirnen aus, kümmern sich um Pflanzen und Außenanlagen oder trennen und entsorgen Abfall. Unter administrative Tätigkeiten fallen beispielsweise das Anfertigen von Kopien oder das Sortieren von Akten und Karteikarten. Der Ausbildungsschwerpunkt wurde bewusst auf die Praxis gelegt, da die Stärken der meisten Jugendlichen in diesem Bereich liegen.

Das Curriculum für den theoretischen Unterricht wurde eigens mit Blick auf die Zielgruppe und auf den Bedarf in der Praxis entwickelt und unterliegt einem kontinuierlichen Revisionsprozess. In der Schule wird vor allem das vermittelt, was die Jugendlichen konkret brauchen, um in der Praxis gut arbeiten zu können, beispielsweise Hintergrundwissen zu alten, behinderten und kranken Menschen. Der richtige Umgang und kreative Beschäfti- 
gungsmöglichkeiten mit Bewohnern und Patienten werden ebenso erlernt wie das Zubereiten von kleinen Mahlzeiten. Wichtige Regeln für Transportdienste, der Umgang mit Werkzeug und Geräten sowie Hygiene-Richtlinien stehen genauso auf dem Stundenplan wie ein Erste-Hilfe-Kurs und grundlegende Kommunikationsregeln zur Stärkung der sozialen Kompetenz.

Wer die Prüfungen nach dem ersten Ausbildungsjahr erfolgreich absolviert, wird bei den jeweiligen beteiligten Trägern für mindestens ein weiteres Praxisjahr angestellt und erhält damit die Chance, sich weiter in der Praxis zu bewähren. Dieses Beschäftigungsjahr ist zentraler Bestandteil des Modellprojekts. Die Ausbildung wird in Baden-Württemberg nach Abschluss des Praxisjahrs staatlich anerkannt. Durch die Ausbildung ist die Berufsschulpflicht abgegolten.

\section{Wie das Projekt entstand}

Ausgangspunkt für die Entwicklung des Modellprojekts war - neben der prekären Situation vieler Hauptschulabsolventen beim Übergang von der Schule in den Beruf - der gestiegene Bedarf an Servicekräften in Einrichtungen des Sozial- und Gesundheitswesens. Dienstleistungen für alte, kranke und behinderte Menschen lassen sich nicht "outsourcen «, dieser Bedarf wird aufgrund des demografischen Wandels in Zukunft steigen.

Vor dem Hintergrund des prognostizierten Fachkräftemangels können es sich Arbeitgeber nicht leisten, auf Teile der jüngeren Generation zu verzichten, sondern sollten das Potenzial von eher praktisch begabten Jugendlichen, die vielleicht keine glänzenden Schulnoten aufweisen, erkennen und fördern. Die Evaluationsergebnisse

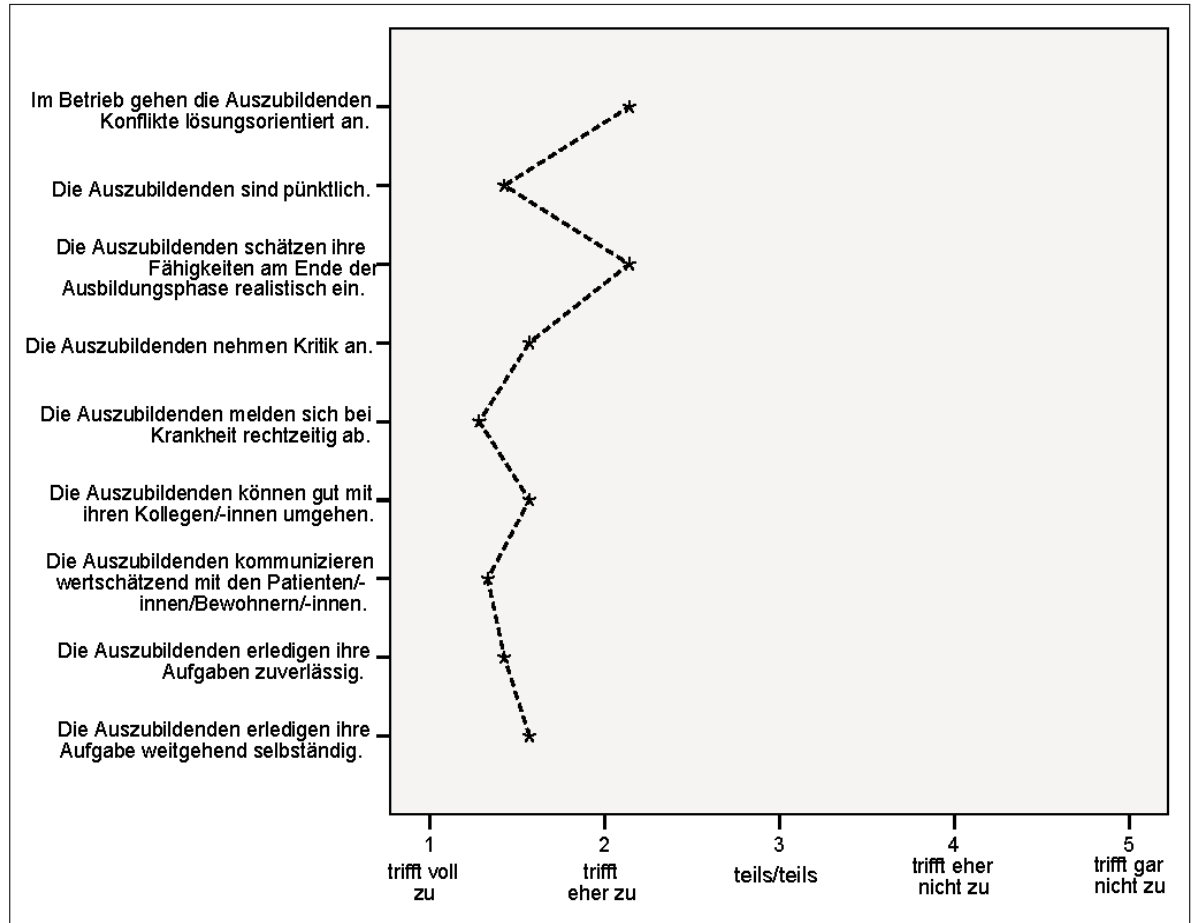

Abb. 1: Einschätzungen der Praxis zu den Kompetenzen der Jugendlichen am Ende des Ausbildungsjahres

Quelle: Univation, Institut für Evaluation

\begin{tabular}{|lccccc|}
\hline & $\begin{array}{c}\text { triff } \\
\text { voll } \\
\mathrm{zu}\end{array}$ & $\begin{array}{c}\text { trift } \\
\text { eher } \\
\mathrm{zu}\end{array}$ & teils/ teils & $\begin{array}{c}\text { trifft } \\
\text { eher } \\
\text { nicht zu }\end{array}$ & $\begin{array}{c}\text { trift } \\
\text { gar } \\
\text { nicht zu }\end{array}$ \\
$\begin{array}{l}\text { Die Ausgebildeten sind gut auf die } \\
\text { Beschäftigungsphase vorbereitet. }\end{array}$ & 3 & 4 & -- & -- & -- \\
$\begin{array}{l}\text { Die Ausgebildeten verfügen über die } \\
\text { notwendigen Kompetenzen, um die } \\
\text { Beschäftigungsphase gut absolvieren zu können. }\end{array}$ & 4 & 3 & -- & -- & -- \\
$\begin{array}{l}\text { Wir gehen davon aus, dass unsere Servicehelfer } \\
\text { die Beschäftigungsphase erfolgreich beenden. }\end{array}$ & 6 & 1 & -- & -- & -- \\
\hline
\end{tabular}

Abb. 2: Einschätzungen der Praxis zu den Kompetenzen der Jugendlichen am Ende des Ausbildungsjahres

Quelle: Univation, Institut für Evaluation des Modellprojekts »Servicehelfer « machen deutlich, dass diejenigen Servicehelfer, denen etwas zugetraut wird und die erleben, wie es ist, sinnvolle Arbeit auszuführen und in den jeweiligen Einrichtungen gebraucht zu werden, zuverlässig und empathisch arbeiten und sich zumeist gut in die Strukturen ihrer praktischen Einsatzstellen eingefunden haben. Arbeitgeber im Sozialund Gesundheitswesen können so einen Beitrag zur beruflichen Integration von Hauptschulabsolventen leisten, der sich für die Einrichtungen lohnt.

\section{Entlastung für Pflegekräfte}

Es ist bekannt, dass gerade in der Pflege häufig eine hohe Arbeitsbelastung vorherrscht und dass ein beträchtlicher Teil der Arbeitszeit für nicht originär der Pflege zuzuordnende Tätigkeiten eingesetzt wird. Durch die Servicehelfer soll hochqualifiziertes Personal von Aufgaben, für die Servicekräfte viel besser qualifiziert und andere überqualifiziert sind, entlastet werden. Gleichzeitig soll mit einem breiteren Angebot an Service und Dienstleistung eine Qualitätsverbesserung in den Einrichtungen einhergehen. Dadurch profitiert der Patient, Bewohner und Kunde, was durch die positiven Reaktionen der Bewohner und Patienten im Verlauf des Modellprojekts deutlich bestätigt wird.

Servicehelfer können in ihrer jugendlich frischen Art entscheidend dazu beitragen, durch qualitativ gut erbrachte sachund personenbezogene Dienstleistungen die Servicequalität in Alten- und Behindertenhilfeeinrichtungen sowie in Kliniken anzuheben. Dies bestätigen erste Aussagen der Träger zu Zufriedenheitsumfragen bei ihren Kunden.

Die Träger, die am Ende des Ausbildungsjahres des ersten Jahrgangs Servicehelfer ausgebildet haben, schätzen die Dienstleistungsqualität in ihren Einrichtungen durchschnittlich um knapp zehn Prozent höher ein als ohne Servicehelfer. Gründe hierfür lagen für sie beispielsweise in der Verkürzung der Wartezeiten bei der Aufnahme im Krankenhaus oder beim Transport zu Untersuchungen und in der umsichtigen und schnelleren Versorgung mit Speisen und Getränken. Gleichzeitig wurden die anderen Mitarbeiter nach Aussage der Träger durchschnittlich um circa elf Prozent entlastet.

Übergeordnetes Ziel des Modellprojekts ist es, den Bedarf an Dienstleistungen im Sozial- und Gesundheitswesen zu decken und dabei die Jugendlichen dauerhaft in den ersten Arbeitsmarkt zu vermitteln. Dies scheint zu gelingen, denn die meisten Servicehelfer des ersten Projektdurchgangs werden nach den ersten beiden 
Jahren bei ihren jeweiligen praktischen Einrichtungen angestellt bleiben. Einige arbeiten weiterhin als Servicehelfer, andere schließen dort eine Ausbildung in der Kranken- oder Altenpflege an. Dies macht deutlich, dass die Jugendlichen ihre Chance genutzt und sich in ihren praktischen Einrichtungen bewährt haben. Gleichzeitig können die Einrichtungen auf diese Weise langfristig Personal rekrutieren und das betriebsspezifische Wissen des Servicehelfers nutzen, das in der Ausbildung erworben wurde.

Die Jugendlichen sind im Modellprojekt "Servicehelfer « persönlich und fachlich gereift und haben wesentliche soziale und berufliche Kompetenzen entwickelt. Dies belegen auch die Angaben von sieben Trägern zum Ende des ersten Ausbildungsjahres (Abb. 1 und 2).

\section{Was Ausstellungsträger leisten müssen}

Ein gelingender Einsatz von Servicehelfern und eine darauf aufbauende nachhaltige Implementierung des Berufsbilds setzen in der Praxis einige Vorarbeit voraus. Je klarer das Aufgabenprofil, die organisatorische Verortung und die Praxisanleitung der Servicehelfer seitens der Einrichtung im Vorfeld definiert sind, desto größer ist die Aussicht auf einen positiven und erfolgreichen Ausbildungsverlauf auf beiden Seiten. Die Evaluationsergebnisse belegen, dass die Einrichtungen, die im Vorfeld intensive konzeptionelle Arbeit geleistet haben, mit ihren Servicehelfern sehr gute Erfahrungen gemacht haben (Abb. 3 und 4).

Die positiven Erfahrungen, die die Jugendlichen gesammelt haben und ihr je-

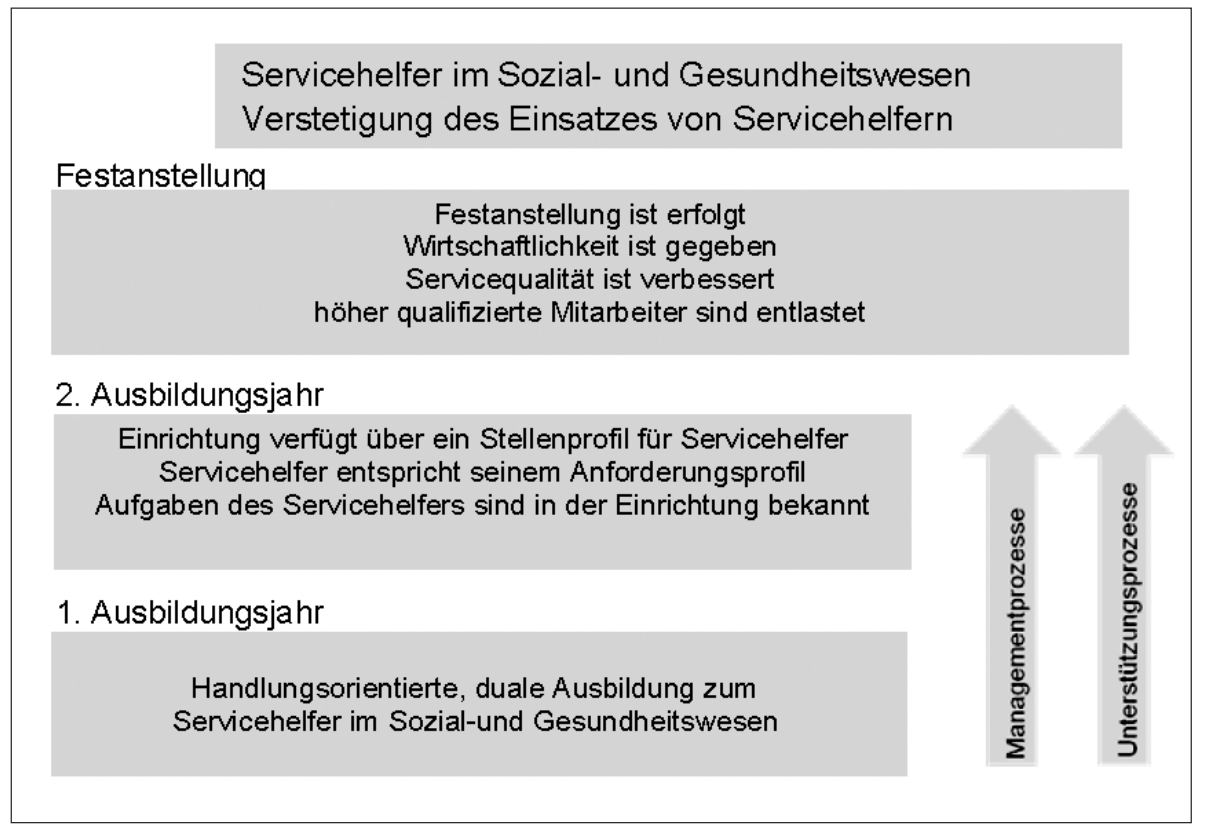

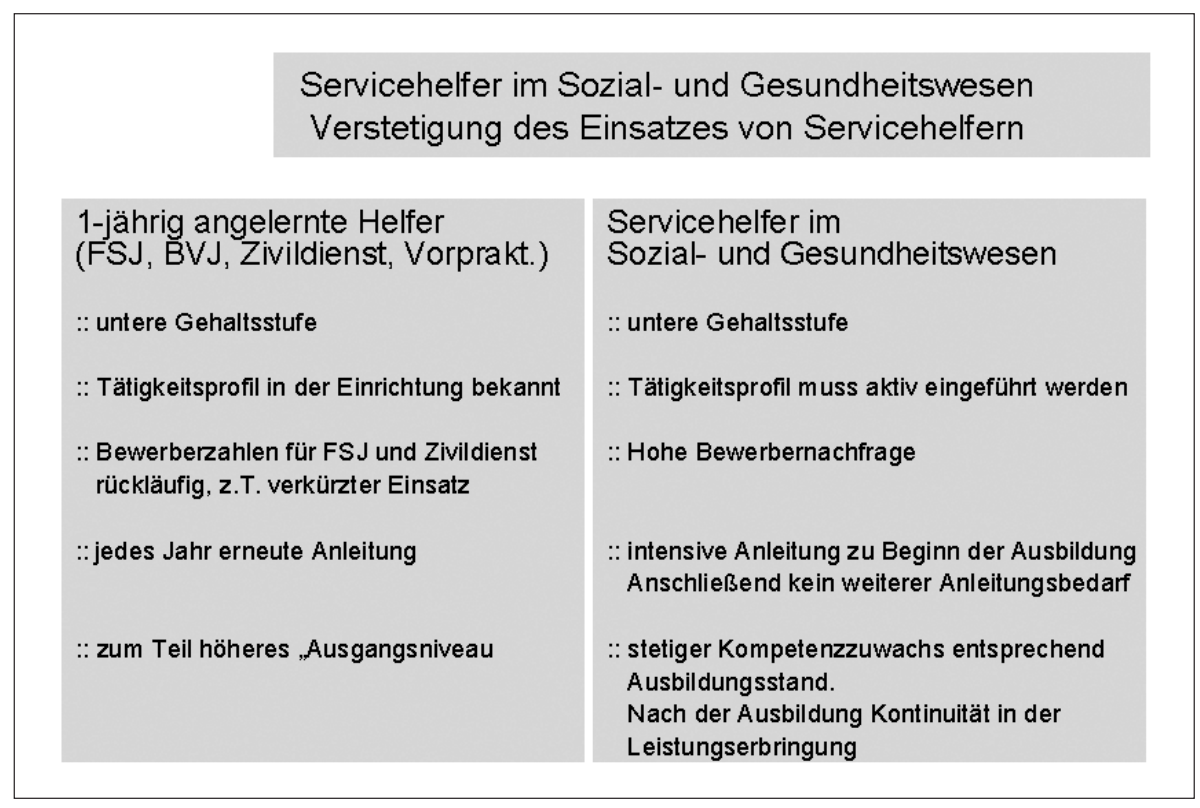

